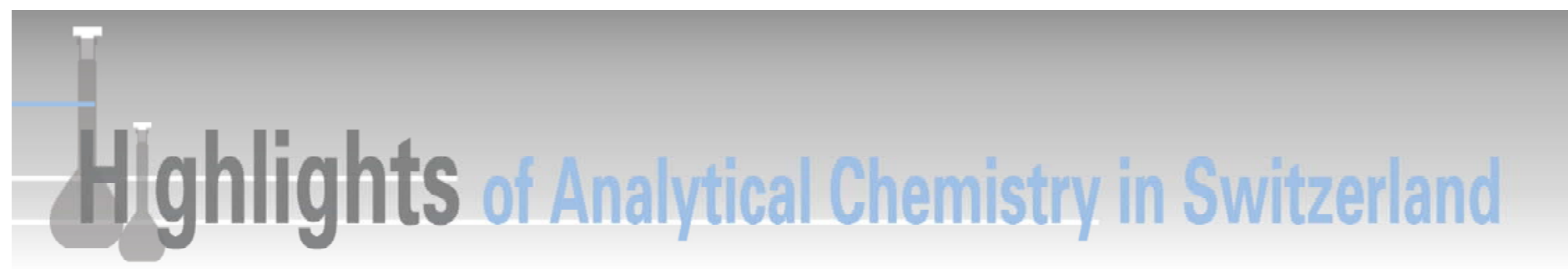

\section{Screening of Samples for the Presence of Chemical Warfare Agents by Nuclear Magnetic Resonance}

\section{Urs C. Meier}

Correspondence: Dr. Urs. C. Meier, Swiss NBC Defence Establishment, Spiez Laboratory, $\mathrm{CH}-3700 \mathrm{Spiez}$

Tel. +4133228 16 92, Fax +413322814 02, E-Mail: urs.meier@babs.admin.ch

Keywords: Chemical warfare agents - Chemical Weapons Convention · Nuclear magnetic resonance spectroscopy · Organophosphorus nerve agents $\cdot$ Sarin

Chemical weapons were first used on a large scale in World War I. At this time the main chemical warfare agents (CWA) were blistering agents (mustard gas) and asphyxiating agents (phosgene). Since the 1930s organophosphorus nerve agents (e.g. sarin, VX) have been synthesized. Public interest in this topic was stressed in the Iran-Iraq conflict (1980-1988) and through a terrorist attack in the Tokyo subway system. In 1995 sarin was released by the Aum sect resulting in 12 deaths and 5000 poisoned or injured people.

On 29 April 1997, the Chemical Weapons Convention (CWC) entered into force. The convention prohibits the development, production, stockpiling and deployment of these weapons. The Organisation for the Prohibition of Chemical Weapons (OPCW) is responsible for the implementations of the CWCs provisions, including verification of its compliance. Ever since, the OPCW has organized interlaboratory proficiency tests to designate laboratories for the verification of the CWC. Spiez Laboratory is one of several designated laboratories worldwide.

Modern analytical methods for the detection and unambiguous identification of CWAs are needed for the verification of the CWC, the protection of the population and the proper medical treatment of victims. The samples to be analysed may be a liquid, soil or shell fragments. After a sample preparation step, they are subjected to instrumental analysis.

Among other techniques, nuclear magnetic resonance (NMR) spectroscopy is suitable for the analysis of CWAs. Traditionally, the screening is done by ${ }^{1} \mathrm{H}$ and ${ }^{31} \mathrm{P}\left\{{ }^{1} \mathrm{H}\right\}$ NMR. ${ }^{1} \mathrm{H}$ NMR is limited by the high level of background present in environmental samples, ${ }^{31} \mathrm{P}\left\{{ }^{1} \mathrm{H}\right\}$ by its low sensitivity and poor information content. Recently, nonselective $1 \mathrm{D}{ }^{1} \mathrm{H}-{ }^{31} \mathrm{P}$ inverse NMR experiments were shown to be the most sensitive NMR methods to selectively screen samples for the presence of nerve agents. Only signals stemming from the nerve agents are detected and the background is completely eliminated. Samples spiked at the $5 \mathrm{ppm}$ level can be screened within an hour, whereas for ${ }^{31} \mathrm{P}\left\{{ }^{1} \mathrm{H}\right\}$ an overnight acquisition is necessary.

\section{Reference}

U.C. Meier, Anal. Chem. 2004, 76, 392.

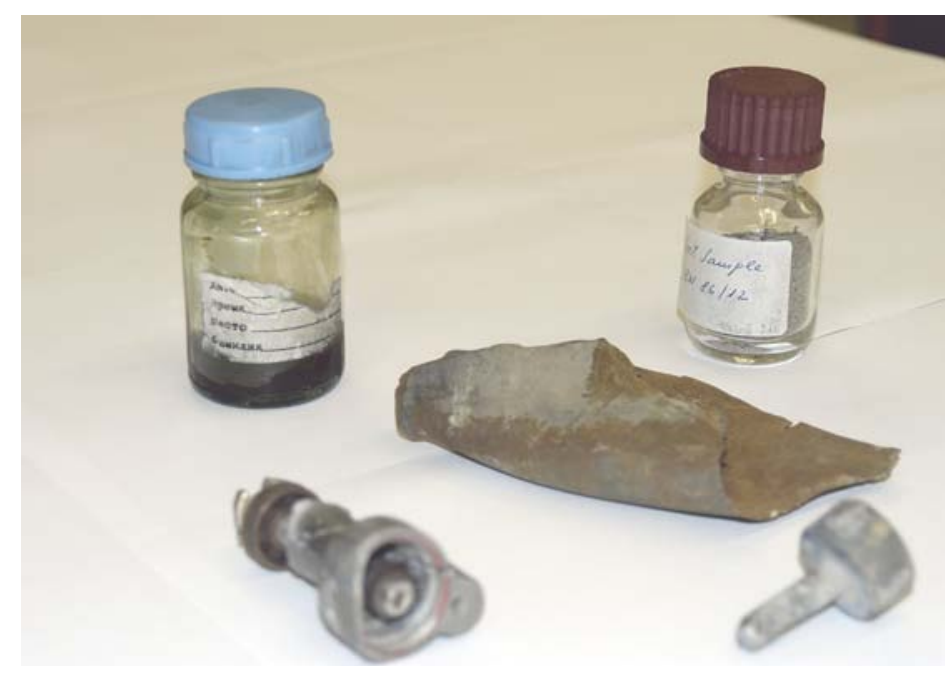

Various types of samples suspect of contamination with chemical warfare agents
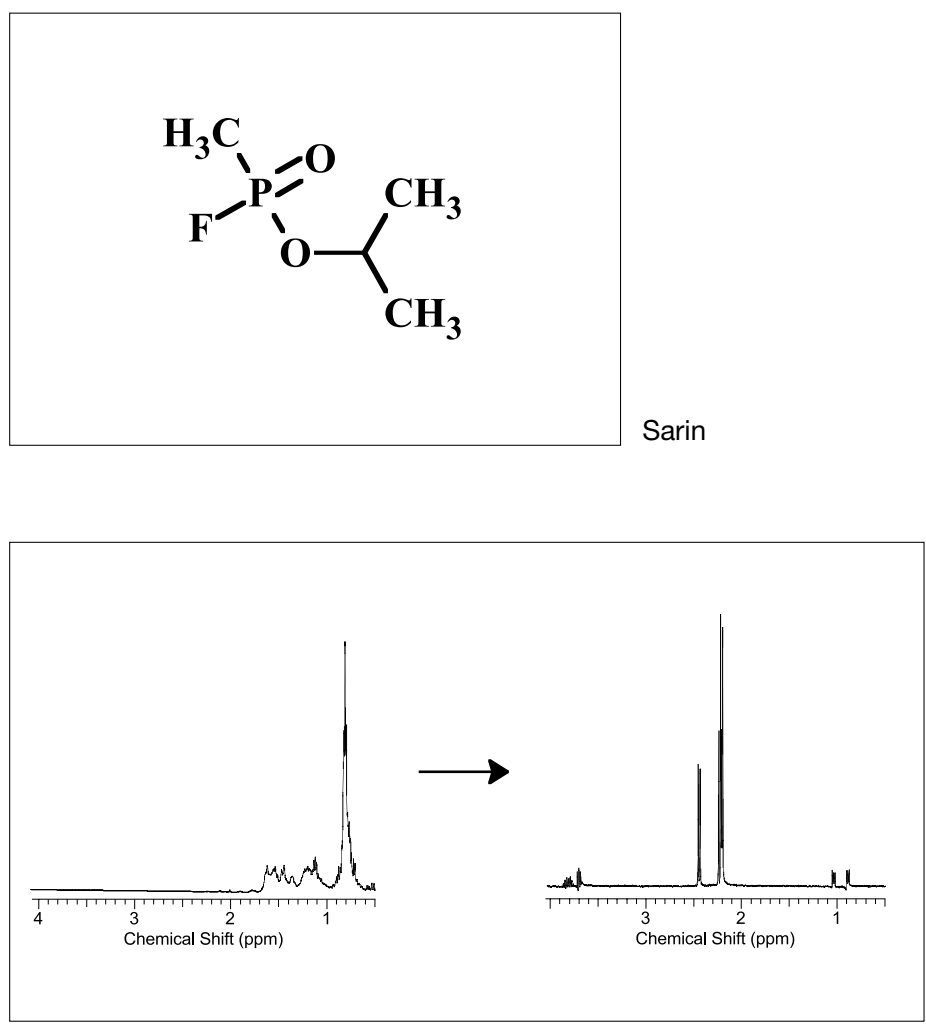

The spectrum on the left shows a standard ${ }^{1} \mathrm{H}$ spectrum, whereas the ${ }^{1} \mathrm{H}$ ${ }^{31} \mathrm{P}$ HSQCTOCSY spectrum of the same solution is shown on the right. The background present in the ${ }^{1} \mathrm{H}$ spectrum is completely eliminated in the ${ }^{1} \mathrm{H}$ ${ }^{31} \mathrm{P}$ HSQTOCSY experiment and the previously hidden signals belonging to organophosphorus compounds are exclusively detected. 